\title{
Producción agrícola intensiva: una estrategia de tecnificación agrícola modelo Almería
}

\author{
Intensive Agricultural Production: A Strategy of Agricultural Technification \\ model Almería
}

Artículo recibido en septiembre 2020

Arbitrado en octubre 2020

Aceptado en octubre 2020

Publicado en enero 2021

\author{
Paola Daniela Soruco Parada \\ sorucoparadadani@gmail.com \\ ORCID: 0000-0001-9133-4804 \\ Universidad Andina Simón Bolívar \\ Sucre - Bolivia
}

Palabras clave

RESUMEN

ABSTRACT

Keywords
A nivel mundial existe preocupación por la capacidad de abastecimiento de la producción agrícola. El objetivo de este artículo científico fue proponer un Modelo de Tecnificación Agrícola de alto rendimiento para el municipio de Sucre (Bolivia) que permita cubrir los requerimientos de productos alimenticios de la región. El estudio asumió un enfoque mixto que consistió en realizar un diagnóstico de la producción agrícola de esta región, para identificar las posibilidades de adecuación del modelo de agricultura intensiva vigente en el municipio de Almería (España). Como resultado se propuso una estrategia de implantación que implica el trabajo sistemático en siete áreas fundamentales: recursos naturales, recursos humanos, infraestructura y equipamiento, innovación tecnológica, capacitación y asistencia técnica, respeto al medio ambiente y cooperación institucional. El documento concluye probando mejoras en el rendimiento de la producción agrícola, mediante un ejemplo teórico referido a la producción de tomate en los distritos 7 y 8 de Sucre.

Invernaderos; productividad; agricultura; tecnificación agrícola; recursos naturales; medio ambiente

Globally, there is concern about the supply capacity of agricultural production. The objective of this scientific article was to propose a high-performance Agricultural Technology Model for the municipality of Sucre (Bolivia) to meet the food requirements of the region. The study took a mixed approach that consisted of making a diagnosis of agricultural production in this region, to identify the possibilities for adequacy of the intensive agriculture model in force in the municipality of Almería (Spain). As a result, an implementation strategy was proposed involving systematic work in seven key areas: natural resources, human resources, infrastructure and equipment, technological innovation, training and technical assistance, respect for the environment and institutional cooperation. The document concludes by testing improvements in the yield of agricultural production, using a theoretical example referring to the Tomato production in the districts 7 and 8 of Sucre.

Greenhouses; productivity; farming; agricultural technification; natural resources; environment 
INTRODUCCIÓN

Las tasas de crecimiento de la producción agropecuaria mundial y los rendimientos de los cultivos han disminuido, suscitando temores de que el mundo no sea capaz de incrementar lo suficiente la producción para asegurar una alimentación adecuada de la población futura (FAO, 2020). En este sentido han surgido algunos modelos productivos que podrían mitigar este problema.

La agricultura intensiva aplicada en el municipio de Almería, España, es un modelo de explotación agrícola de alto rendimiento técnico y económico basado en el empleo racional del agua, el enarenado, el uso de invernaderos de plástico, elevada capacitación técnica y alto nivel de empleo de insumos, sobre las características peculiares del medio, con un clima de baja precipitación pluvial (UCM,2020).

En Bolivia, específicamente en las zonas de producción agrícola de la ciudad de Sucre, se tiene condiciones climáticas parecidas a las zonas agrícolas de Almería, lo que permite replicar sus sistemas de producción y adaptarlos, obteniendo resultados similares. Si bien las condiciones de producción en esta ciudad son tradicionales, el contexto es propicio para aplicar la tecnología de agricultura intensiva. Según la FAO (2020), Sucre cuenta con diversas iniciativas y colectivos que impulsan la producción de alimentos saludables.

A partir del año 2008, gracias a un convenio entre la Universidad Andina Simón Bolívar (UASB) Sede Bolivia y la Junta de Andalucía (España), se ha permitido a varios técnicos y ejecutivos Bolivianos visitar zonas productivas de España bajo el modelo de producción intensiva en invernaderos tecnológicamente efectivos, a su vez, bajo el mismo convenio se ha instalado un invernadero en la ciudad de Sucre en el Campus de la UASB con excelentes resultados productivos, constituyéndose como modelo demostrativo de que este tipo de producción es altamente efectiva para la región (UASB, 2008).

De igual manera, siguiendo esta línea de producción, la Gobernación del departamento de Chuquisaca se encuentra ejecutando un proyecto de Huertos Familiares en la ciudad de Sucre, el mismo que permite a varias familias contar con vegetales frescos para su alimentación y comercializar el excedente de su consumo. Los productos encuentran mercado rápidamente, ya que se conoce que este método de producción otorga cualidades especiales (GADCH, 2018).

$\mathrm{Si}$ bien estos huertos soy exitosos, equivalen a una muy pequeña parte de la producción necesaria para alimentar a la población local. El municipio se abastece de hortalizas de otros departamentos y países (Tarija, Cochabamba, Santa Cruz, Potosí, Argentina, Chile y Perú), debido a que la producción agrícola tradicional local utiliza métodos con bajos rendimientos y baja productividad. En términos generales, existe un limitado acceso a alimentos variados, sanos y nutritivos, así como inadecuadas conductas y hábitos alimenticios, y por consiguiente malnutrición (desnutrición, anemia, sobrepeso obesidad) en la población (SITAP, 2015). 
En este contexto, el objetivo de este artículo científico fue proponer un Modelo de Tecnificación Agrícola de alto rendimiento para el municipio de Sucre que permita cubrir los requerimientos de productos alimenticios de la región. Los apartes del documento son: diagnóstico de las potencialidades de producción existentes en el municipio de Sucre; construcción de una estrategia de producción tecnificada proyectada a 5 años; resultados previstos para el rendimiento y calidad de la producción agrícola (se tomó como ejemplo la producción de tomate).

METODOLOGÍA

La investigación se desarrolló bajo un enfoque mixto de corte transversal y alcance descriptivo. El estudio se desarrolló en tres etapas, las dos primeras se desarrollaron con enfoque cualitativo y la última requirió un enfoque cuantitativo.

Se partió por el estudio de las potencialidades de producción existentes en el municipio de Sucre. En esta etapa se aplicó el método bibliográfico para conocer los resultados de estudios previos que han caracterizado de alguna manera la producción agrícola en el municipio de Sucre. Los estudios que se consideraron fueron los presentados por GAMS (2015) y la FAO (2020). Para fortalecer el diagnóstico fue necesario recoger información de fuentes primarias por medio de una entrevista estructurada dirigida a autoridades del Municipio. Las autoridades consultadas del Gobierno Autónomo Municipal de Sucre (GAMS) fueron: Secretario de Planificación, Secretario de Desarrollo Productivo, Director de Desarrollo productivo y
Jefe de Producción. Asimismo, se conversó con siete Subalcaldes de los diferentes distritos del municipio y los representantes los distritos rurales Centrales y Subcentrales. El objetivo de la entrevista fue conocer la problemática de producción agrícola en Sucre.

La segunda etapa del estudio consistió en la construcción de una estrategia de producción tecnificada proyectada a 5 años. Para esto se tomó como base la experiencia exitosa presentada por Valera et al. (2000) del modelo de producción implementado en el municipio de Almería (España). Se propuso una adecuación que implica el trabajo sistemático en siete áreas estratégicas fundamentales: recursos naturales, recursos humanos, infraestructura y equipamiento, innovación tecnológica, capacitación y asistencia técnica, respeto al medio ambiente y cooperación institucional.

Finalmente, en la tercera etapa se tomó como ejemplo la producción de tomate y se demostró que implementar una estrategia de producción agrícola tecnificada eleva el rendimiento y calidad de la producción.

\section{RESULTADOS}

El trabajo se realizó en tres etapas, la primera se constituye en un diagnóstico de las potencialidades de producción existentes en el municipio de Sucre, la segunda estuvo destinada a la construcción de una estrategia de producción tecnificada proyectada a 5 años. Finalmente, en la tercera etapa se presentan los resultados previstos para el rendimiento $\mathrm{y}$ calidad de la producción agrícola en la ciudad de Sucre, tomando como ejemplo la producción de tomate. 


\section{Potencialidades de producción}

Los métodos productivos tradicionales aplicados en Sucre implican altos costos y baja productividad, sin proyecciones claras hacia el vertiginoso avance tecnológico mundial en el área de producción de alimentos. En este municipio, tomando los análisis realizados por el GAMS (2015) y la FAO (2020), existen dos zonas productivas con condiciones ambientales apropiadas para la producción agrícola, la región de Quila Quila y la zona de influencia del Rio Chico (El Valle). Si bien estas zonas están diferenciadas en su localización, ambas son altamente productoras de hortalizas y tienen la misma problemática en los procesos productivos, relacionada con el uso de tecnología tradicional, que se puede caracterizar de la siguiente manera: (a) labranza manual con tracción animal, en casos esporádicos con maquinaria, (b) uso de semilla local, en muchas ocasiones semilla propia, (c) riego por surco con una baja eficiencia en el uso del agua, (d) bajo uso de insumos como fertilizantes y agroquímicos nocivos para el medio ambiente, (e) mano de obra familiar y en pocas etapas contratada, ( $f$ ) baja asistencia técnica, $(\mathrm{g})$ cultivos estacionales, es decir todos cosechan en la misma época, provocando incrementos estacionales de la oferta $y,(h)$ métodos de almacenaje y transporte ineficientes.

Si bien en el último Censo Agropecuario (INE, 2015), el municipio tiene en producción 12.588 hectáreas y cuenta con 17.747 personas que trabajan en la agricultura, según entrevistas a las autoridades de los distritos rurales del municipio (Subalcaldes, centrales, subcentrales, dirigentes campesinos), en los últimos 5 años se ha manifestado un incremento de las familias que tienen como estrategia de vida el comercio y el trasporte. Por lo que se considera que en los distritos 7 y 8 de este municipio, aproximadamente existen 12.000 agricultores.

En estos distritos, según el estudio de campo realizado, se identificó la siguiente problemática: (a) Una baja productividad a consecuencia del uso de tecnología tradicional; (b) Los productores están en una situación de desventaja frente a la producción foránea, se ven entonces obligados a bajar los precios a un nivel que apenas cubre los costos de producción; (c) Esta situación reduce las posibilidades de inversión en tecnología, es decir, no se cuenta con ahorros para invertir en semillas mejoradas y agroquímicos actualizados, dejando la ocupación de los terrenos para una producción frutícola sin normas técnicas adecuadas; (d) El criterio para escoger los agroquímicos está basado en el precio, sin embargo no se considera el cuidado al medio ambiente y a la salud; (e) El riego es por sistema de surco, lo cual deriva en una mala utilización del líquido elemento; (f) En esta zona no existen posibilidades de almacenamiento de la producción, por lo que se cosecha para la venta inmediata; (g) El apoyo interinstitucional a los productores del municipio es bajo, limitándose a cubrir las pérdidas por emergencias, como fenómenos climáticos adversos, riadas; (h) No existe la capacitación técnica necesaria que pueda mejorar las condiciones de la producción.

De acuerdo con la experiencia local en producción urbana $(\mathrm{GADCH}, 2018)$ y la revisión de la experiencia de Almería (Urresterazu, 
2000), es posible aplicar un tipo invernadero que integre ambos escenarios y se adapte a la situación local, considerando variaciones solamente en cuanto al material (plástico) y su forma: invernadero tipo túnel, por ser de fácil construcción, bajo precio y porque requieren insumos que se encuentran en el mercado local.

\section{Estrategia de producción tecnificada}

Con base en la realidad expuesta, se plantea la estrategia de producción agrícola tecnificada que tiene por objetivo obtener una producción de alto rendimiento y calidad en el municipio de Sucre, que permita cubrir los requerimientos de productos alimenticios de la región. Se estima que en la primera etapa de implementación (5 años) es posible cambiar el tipo de producción del $10 \%$ de los productores de los distritos 7 y 8 (1.200 actores sociales beneficiados directamente).
Los objetivos específicos que persigue esta estrategia son: incrementar la inversión en la agricultura, diversificar la producción, definir progresos técnicos, mejorar la calificación de la mano de obra, fortalecer la base productiva, incrementar la productividad agrícola, desarrollar sistemas de capacitación y estimular la participación tanto de organizaciones no gubernamentales como de instituciones públicas y privadas.

Según se presenta en la Figura 1 , la estrategia implica un trabajo sistemático enmarcado en siete áreas fundamentales de acción: recursos naturales, recursos humanos, infraestructura y equipamiento, innovación tecnológica, capacitación y asistencia técnica, respeto al medio ambiente y cooperación institucional.

\section{Figura 1}

Estrategia de producción agrícola tecnificada

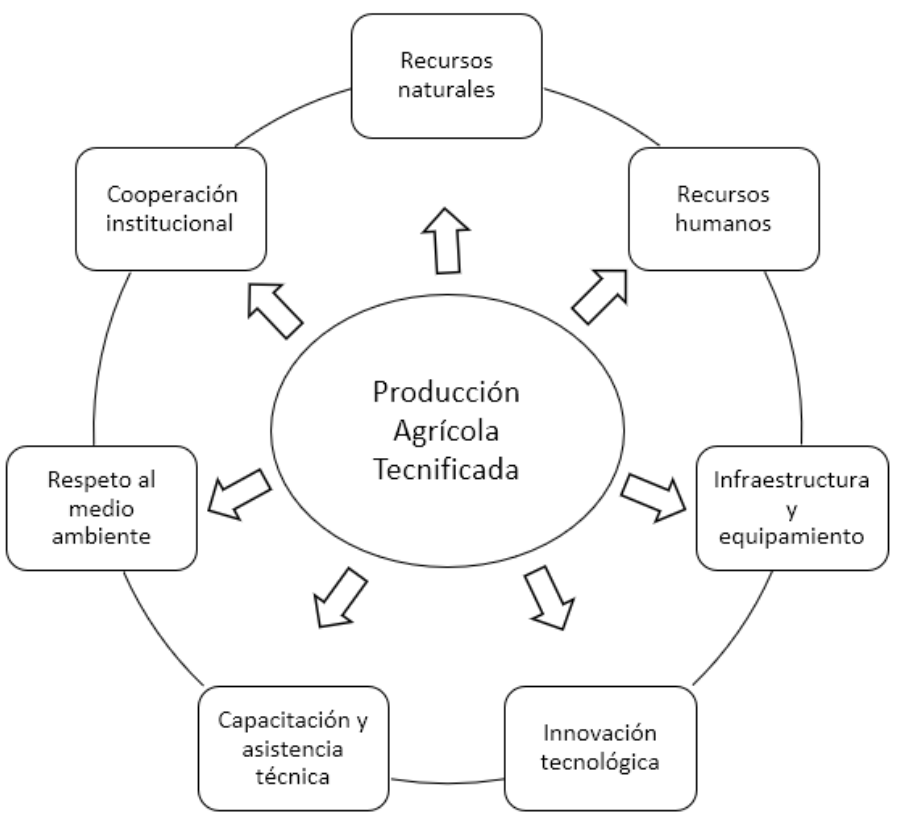


La interrelación de los elementos expuesto en la Figura 1 permitirá elevar la calidad y la productividad agrícola. Por tanto, estos siete componentes se constituyen en las bases estratégicas sobre las que se deberá actuar para elevar el nivel de ingresos y rentabilidad de los actores sociales (agricultores e inversores).

\section{Innovación tecnológica}

La innovación tecnológica consiste en implementar invernaderos tipo túnel en la producción agrícola con el objeto de crear ambientes especiales que generen condiciones favorables y permitan el incremento de la producción. De la misma manera, aseguren la obtención de productos evitando los fenómenos adversos. Se debe aplicar tecnología actualizada con base en investigaciones externas (Almería) y locales (Agricultura urbana) que mejoren la calidad organoléptica y nutricional de esta producción.

Estos invernaderos deben ubicarse en sitios donde exista la provisión de agua permanente, es decir, que en el municipio de Sucre deberán ubicarse en Quila Quila (Distrito 8) y El Valle (Distrito 7). Estas zonas cumplen los requisitos óptimos relacionados con clima y suelo: (a) temperatura adecuada sin saltos térmicos importantes que limiten la producción de hortalizas (SENAMHI,2020); (b) altura sobre el nivel del mar apropiada, que aporta con los niveles de oxígeno y dióxido de carbono necesarios para la producción agrícola; (c) duración del día mayor a las doce horas, para evitar el uso de luz artificial; (d) suelo agrícola con textura, estructura, profundidad y fertilidad apropiados, materia orgánica que no afecta el desarrollo de los cultivos.

Habiendo corroborado el cumplimiento de los requisitos anteriores, se debe desarrollar las siguientes actividades: (1) Definir los elementos del clima que permitan la zonificación agroecológica necesaria; (2) Definir el nivel tecnológico y las necesidades de capacitación para el recurso humano.

\section{Respeto al medio ambiente}

Un aspecto fundamental para el desarrollo de esta estrategia fue el respeto al medio ambiente, considerando el valor que tiene la naturaleza, los animales, plantas y todo aquel ser vivo en el planeta. Por lo que se deberá valorar de manera anticipada el impacto ambiental que provocaría este tipo producción, promover el respeto y la protección como valores que deberán ser reflejados en todas las acciones. Para poder intervenir en el entorno particular se debe tomar conciencia de las consecuencias que tiene una gestión adecuada de recursos naturales y los efectos inmediatos y positivos que pueden tener la contribución profesional.

Entre las acciones a desarrollar se identifican las que tienen relación con el medio ambiente: (1) Se trata de introducir techos de plástico cuya vida útil tiene una duración entre 5 y 10 años, por lo que hay que prever su desecho en zonas permitidas o hacer un tratamiento para evitar el impacto negativo de este material; (2) Se trata de utilizar todos los agroquímicos permitidos por la Organización Mundial de la Salud, que no son nocivos para los humanos y son amigables con el medio 
ambiente. (3) El uso del agua debe ser lo más eficiente posible, mediante sistemas presurizados; (4) Todos los desechos orgánicos deben tener un proceso de compostaje que permita su reutilización; (5) Se debe lograr una máxima eficiencia en el uso de la energía; (6) Ubicar los invernaderos en sitios que no afecten a la vegetación nativa, es decir, en terrenos agrícolas antiguos.

\section{Recursos naturales}

Actualmente, una estrategia productiva de los agricultores es la asociación de la producción agrícola con la cría de cabras y vacunos, con un sistema pecuario trashumante. En el caso de las cabras, se las encierra en la noche para obtener el estiércol, para luego guiarlas al cerro durante todo el día. Esta forma de crianza impide la regeneración nativa de la flora y el suelo es erosionado de manera acelerada.

Se considera que la implementación de invernaderos favorecerá la preservación de los recursos naturales, los agricultores invertirán recursos financieros y humanos en las labores agrícolas $y$, como consecuencia, se reducirá la inversión en la cría de animales. Esta disminución en la presión sobre los recursos naturales permitirá una mayor regeneración natural del bosque y sotobosque que provocará una mayor cobertura vegetal protectora del suelo. Asimismo, esta cobertura adicional proveerá de alimento a la fauna silvestre, evitando daño a los cultivos, esta cobertura vegetal a su vez mejorará la formación de suelo en la zona donde se almacena agua, permitiendo contar con fuentes de agua como son los pugios y/o ojos de agua.

Por otra parte, la capacitación técnica deberá ser un medio para formar valores de respecto y preservación del medio ambiente. Esta educación creará las bases en la conciencia de los agricultores para la futura inversión en proyectos de manejo de los recursos naturales.

\section{Infraestructura y equipamiento}

Se propone trabajar con dos tamaños de invernadero: uno pequeño de $500 \mathrm{~m} 2$ útiles, en adelante "Invernadero A", y otro mayor de $5000 \mathrm{~m} 2$ útiles, en adelante "Invernadero B". Se consideró que la combinación de ambos invernaderos permitirá el involucramiento de: a) los agricultores pequeños de todo el municipio que, mediante una inversión pequeña, tendrán la oportunidad de involucrarse en el mejoramiento tecnológico; b) aquellas personas que, contando con capitales mayores, quieran invertir capitales en la producción agrícola tecnificada.

Los métodos constructivos de cada invernadero son idénticos, diferenciándose en el número de túneles que conformarán cada uno. Ambos con la misma cubierta de plástico agrofilm especial que permite el paso de los rayos solares benéficos y evita el escape de calor, favoreciendo de esta manera el desarrollo del cultivo.

Siguiendo criterios principalmente económicos, se propone usar invernaderos tipo túnel, para ambos casos, debido a que cuentan con características que se ajustan a las necesidades del municipio: a) construcción barata y sencilla, b) especialmente diseñado 
para superficies pequeñas y cultivos como hortícolas de porte rastrero o entutorados a baja altura, c) mayor capacidad de estanqueidad que el invernadero plano, d) estructura simple y resistente, e) reduce el problema de la condensación y el goteo del agua en los cultivos debido a la cubierta curva $y$, f) montaje rápido y sin soldaduras.

Este tipo de invernadero no tiene paredes rectas, siendo la estructura totalmente curva desde el punto de fijación en el suelo hasta la cumbrera. Los arcos tienen forma curva. Está compuesto por uno o varios módulos según la forma del terreno y el tamaño del establecimiento. La construcción requiere de una serie de arcos fabricados con tubos cilíndricos galvanizados, los cuales no precisan de zapatas de hormigón, lo que posibilita su traslado y fácil instalación.

\section{Figura 2}

\section{Estructura del invernadero propuesto}

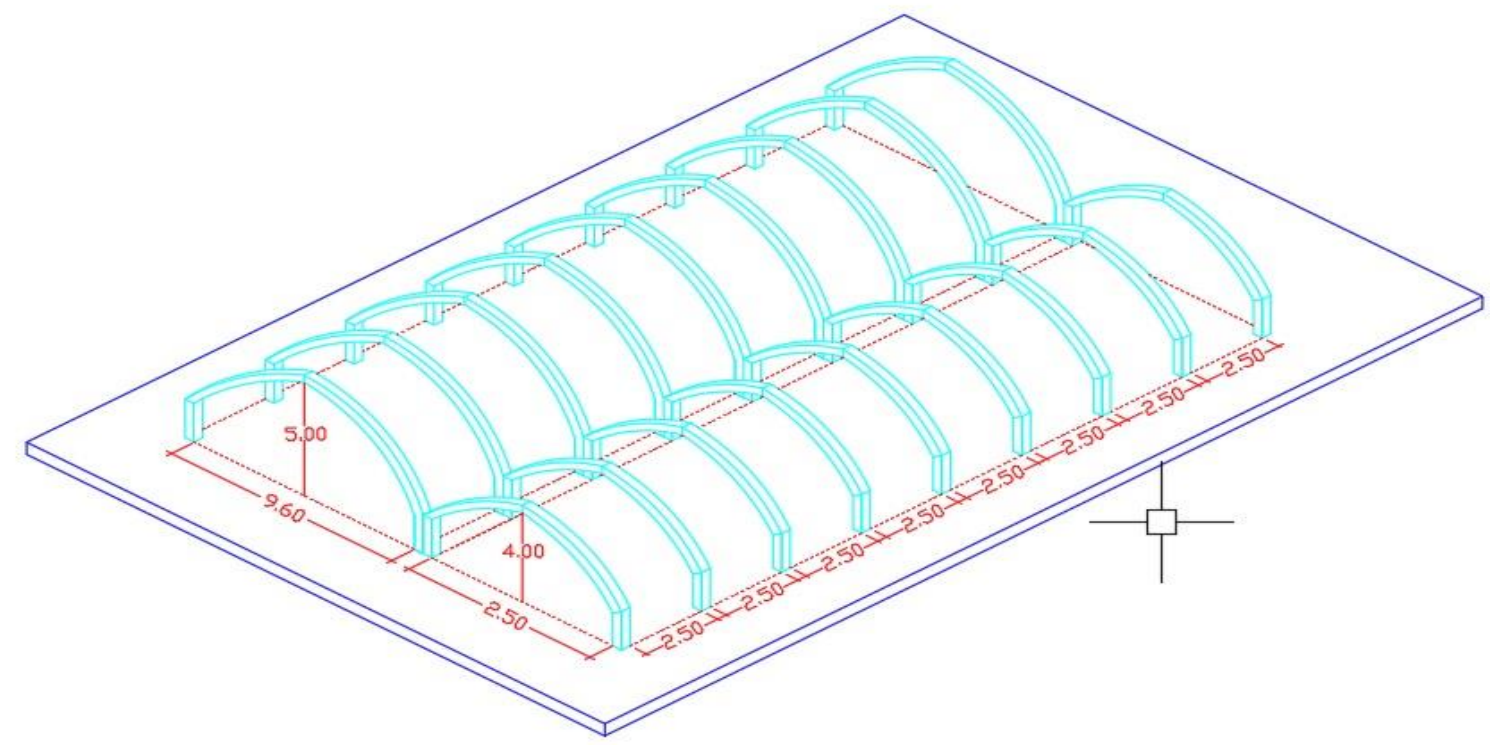

Las dimensiones propuestas para el invernadero A son: $8 \mathrm{~m}$ de ancho, $4 \mathrm{~m}$ de alto, $2.5 \mathrm{~m}$ de distancia externa entre arcos, se considera bastidores de refuerzo perimetrales. Se estima que para este tipo de invernadero se requerirán dos módulos de 32 metros de largo (según se muestra en la Figura 2), sin embargo, depende de la forma del terreno.

Las dimensiones propuestas para el invernadero $\mathrm{B}$ son: $8 \mathrm{~m}$ de ancho, $4 \mathrm{~m}$ de alto, $2.5 \mathrm{~m}$ de distancia externa entre arcos, se considera bastidores de refuerzo perimetrales. Se estima que se requerirán 10 módulos de 63 metros de largo, sin embargo, dependerá de la forma del terreno. 
Existen actividades comunes para los Invernaderos tipo $A$ y $B$, como son las siguientes: 1) Construcción de arcos, pilares, correas, barras, canales, esquineros y refuerzos en taller; 2) Nivelado de la superficie donde se instala, conforme a los criterios de evacuación de agua e inclinaciones; 3) Limpieza y adecuación del terreno a las labores propias del movimiento de tierra; 4) Diseño y la construcción de desmontes y/o terraplenes que permitan adecuar la superficie a las restricciones de nivelación (este paso sólo será necesario cuando la superficie donde se instala el invernadero sea demasiado irregular); 5) Replanteo y preparación de la cimentación; 6) Excavación mediante barrenas; 7) Premontaje en obra; 8) Recepción de materiales en obra para su posterior uso. 8) Tratamiento anticorrosivo en la superficie en contacto con la zapata de los pilares mediante alquitrán.

\section{Cooperación institucional}

Se considera fundamental propiciar asociaciones estratégicas entre los centros de investigación científica y tecnológica y las organizaciones, con el objetivo de desarrollar espacios de participación de las instituciones y los agricultores. Asimismo, estos sistemas de producción deben lograr el esfuerzo institucional para superar los problemas que se presenten en los procesos productivos.

Esta estrategia requiere de la inversión pública, destinada a fomentar la innovación tecnológica del aparato productivo del municipio, de tal forma que se libere esfuerzos para el agricultor.
La estrategia propuesta plantea las siguientes líneas de acción: 1) Demostrar la eficiencia tecnológica de los invernaderos para fomentar la inversión privada; 2) Participación de jóvenes en competencias tecnológicas, empresariales y productivas; 3) Fortalecimiento de las capacidades de los productores e identificar y canalizar sus demandas de innovación tecnológica. 4) Adecuar y adaptar las herramientas, máquinas agrícolas de acuerdo con los requerimientos. 5) Masificar los programas de formación científica y tecnológica. 6) Establecer convenios con los municipios y el departamento de Chuquisaca para la cofinanciación en la compra de maquinaria agrícola.

Para el cumplimiento de estas líneas de acción se considera necesario: a) Establecimiento de redes de cooperación interinstitucional con las universidades y las Organizaciones sin Fines de Lucro (ONG) de la región, para el fortalecimiento productivo en mejores técnicas de producción agrícola; b) Celebración de convenios con ONGs y entidades públicas para realizar capacitaciones en desarrollo empresarial y productivo; c) Formar y consolidar alianzas estratégicas con productores de la región con el fin de aprovechar sus fortalezas y transferirlas gradualmente a las organizaciones comunitarias.

El inicio de este proceso implicará tres actividades: 1) Participación regional en el diseño de mecanismos para compartir información vía foros de debate, cursillos, seminarios y otros; 2) Relación con otros 
sectores para intercambio de experiencias en materia de agricultura; 3) Difusión y aplicación de pautas para mejorar la competitividad en la productividad del trabajo.

\section{Capacitación y asistencia técnica}

La capacitación técnica de la presente propuesta pretende llevarse a través de dos métodos fundamentales: a) Cursos participativos teórico - prácticos con apoyo de cartillas intercomunales y pasantías durante todo el proceso productivo, visitando los invernaderos y observando que exista mayor dedicación y conocimiento en el proceso de producción. b) Capacitación horizontal, es decir, buscar que sean técnicos capacitados los que intervengan como productores, y sean ellos los que demuestren las bondades de la nueva tecnología.

La capacitación formal o intensiva deberá realizarse sistemáticamente y estar dirigida a los actores locales. El componente teóricopráctico permitirá adquirir competencias (conocimientos, habilidades, destrezas, valores y actitudes), necesarias para optimizar y desempeñar ocupaciones y responsabilidades relacionadas con las siguientes áreas: preservación de los recursos naturales, construcción de infraestructura, preparación del terreno, siembra, fertilización, riego, control de malezas, control de plagas, control de enfermedades y cosecha.

Los fundamentos necesarios para desarrollar la capacitación son: producir conocimientos, introducir conocimientos en la práctica y transformación de la práctica. La mejora en las capacidades de los productores locales debe estar en sintonía con los objetos de trabajo y la participación de las personas interesadas.

\section{Los resultados previstos para la producción tecnificada en el municipio de Sucre}

Habiendo representado las necesidades de los sectores productivos en el municipio de Sucre y desarrollado las bases estratégicas para la producción tecnificada, el aporte se materializa así: más y mejor inversión en la agricultura, diversificación de la producción, incorporación de progreso técnico, cualificación de la mano de obra y fortalecimiento de la base productiva, incremento en la productividad agrícola, implementación de un sistema apropiado de capacitación, incentivos a iniciativas y participación.

Se presenta un cálculo del costo de inversión que tomó en cuenta las dimensiones, materiales y tipo de invernadero expuestos previamente. En la Tabla 1 se presenta un presupuesto general para cada tamaño de invernadero propuesto, con base en invernaderos tipo túnel, el mismo tiene como componentes cerchas metálicas de tubería, cubierta de polietileno de diferentes colores y el equipo tecnológico que considera un precio global. 


\section{Tabla 1}

Presupuesto general Invernaderos tipo $A$ y $B$

\begin{tabular}{lcccc}
\hline \multicolumn{1}{c}{ Descripción } & Unidad & Cantidad & $\begin{array}{c}\text { Costo } \\
\text { unitario }\end{array}$ & Costo total \\
\hline $\begin{array}{lcccc}\text { Invernadero tipo A } \\
\text { Cercha metálica }\end{array}$ & pieza & 25 & 534.75 & 13368.75 \\
$\begin{array}{l}\text { Cubierta de polietileno } \\
\text { Equipo tecnología agrícola }\end{array}$ & $\mathrm{m} 2$ & 689.38 & 24.5 & 16889.81 \\
$\begin{array}{l}\text { Presupuestado total Invernadero tipo A } \\
\text { Invernadero tipo B }\end{array}$ & 1 & 17500 & 17500 \\
$\begin{array}{l}\text { Cercha metálica } \\
\text { Cubierta de polietileno }\end{array}$ & pieza & 250 & 534.75 & $\mathbf{4 7 7 5 8 . 5 6}$ \\
$\begin{array}{l}\text { Equipo tecnología agrícola } \\
\text { Presupuestado total Invernadero tipo B }\end{array}$ & global & 6893.8 & 24.5 & 133687.5 \\
\hline
\end{tabular}

Se estima que el nivel de ingreso de los productores agrícolas se incrementará tras la implementación de la estrategia. Este incremento está en función de los esfuerzos realizados por los propios agricultores. En la
Tabla 2 se presentan los valores promedio de la producción de tomate actuales y los valores de producción de tomate estimados en un escenario de implementación de la estrategia.

\section{Tabla 2}

Incremento en el rendimiento del cultivo del tomate bajo Invernaderos $A$ y $B$

\begin{tabular}{|c|c|c|c|c|c|}
\hline \multicolumn{3}{|c|}{ Producción y rendimiento actual } & \multicolumn{3}{|c|}{$\begin{array}{l}\text { Producción y rendimiento con la } \\
\text { implementación de la estrategia }\end{array}$} \\
\hline $\begin{array}{c}\text { Superficie } \\
\text { cultivada }\left(\mathbf{m}^{2}\right)\end{array}$ & $\begin{array}{c}\text { Volumen de } \\
\text { producción } \\
\text { (kg) }\end{array}$ & $\begin{array}{c}\text { Rendimiento } \\
\text { promedio en } \\
\text { kg/ha }\end{array}$ & $\begin{array}{c}\text { Superficie } \\
\text { cultivada } \\
\left(\mathbf{m}^{2}\right)\end{array}$ & $\begin{array}{c}\text { Volumen de } \\
\text { producción } \\
(\mathbf{k g})\end{array}$ & $\begin{array}{c}\text { Rendimiento } \\
\text { promedio } \\
\text { en } \mathbf{k g} / \mathrm{ha}\end{array}$ \\
\hline 500 & 600 & 12000 & 500 & 2500 & 50000 \\
\hline 5000 & 6000 & 12000 & 5000 & 25000 & 50000 \\
\hline
\end{tabular}

El lado izquierdo de la Tabla 2 muestra el nivel de producción actual para $500 \mathrm{~m}^{2}$ y 5000 $\mathrm{m}^{2}$, con base en un promedio gracias a la recolección de información primaria en los distritos 7 y 8 del municipio de Sucre. En el lado derecho de la tabla, se presenta un nivel de producción futura con la implementación de la estrategia de producción agrícola tecnificada, estos niveles son conservadores frente a la alta producción registrada en trabajos experimentales (UASB, 2016). Considerando que a nivel experimental la producción es en pequeña escala, y que se obtienen diferentes incidencias en un cultivo a gran escala, se ha tomado como base un $40 \%$ del rendimiento obtenido en la experiencia de la UASB. 
En la Tabla 3 se muestra los costos en que incurrirán los agricultores para los dos tipos de invernaderos propuestos en la producción agrícola.

\section{Tabla 3}

Costo de la producción tradicional de tomate (en BOB)

\begin{tabular}{|c|c|c|c|c|c|c|c|}
\hline \multirow[b]{2}{*}{ Detalle } & \multirow[b]{2}{*}{ unidad } & \multicolumn{3}{|c|}{ Invernadero tipo A } & \multicolumn{3}{|c|}{ Invernadero tipo B } \\
\hline & & cantidad & $\begin{array}{c}\text { costo } \\
\text { unitario }\end{array}$ & $\begin{array}{l}\text { costo } \\
\text { total }\end{array}$ & cantidad & $\begin{array}{c}\text { costo } \\
\text { unitario }\end{array}$ & $\begin{array}{l}\text { costo } \\
\text { total }\end{array}$ \\
\hline \multicolumn{8}{|l|}{ INSUMOS } \\
\hline Semilla & onza & 1 & 46 & 46 & 10 & 46 & 460 \\
\hline Insecticida & lit & 1 & 47 & 47 & 10 & 47 & 470 \\
\hline Fungicida & lit & 1 & 150 & 150 & 10 & 150 & 1500 \\
\hline Estiércol & $\mathrm{kg}$ & 300 & 0.617 & 185.1 & 3000 & 0.617 & 1851 \\
\hline Subtotal & & & & 428.1 & & & 4281 \\
\hline \multicolumn{8}{|c|}{ PREPARACIÓN DEL TERRENO } \\
\hline Incorporación abono & Jornal & 2 & 75 & 150 & 20 & 75 & 1500 \\
\hline Remoción & Jornal & 2 & 75 & 150 & 20 & 75 & 1500 \\
\hline Formado de camellones & Jornal & 1 & 75 & 75 & 10 & 75 & 750 \\
\hline Subtotal & & & & 375 & & & 3750 \\
\hline \multicolumn{8}{|l|}{ PLANTACIÓN } \\
\hline Almaciguera & Jornal & 1 & 75 & 75 & 10 & 75 & 750 \\
\hline Repique & Jornal & 1 & 75 & 75 & 10 & 75 & 750 \\
\hline Refallo & Jornal & 1 & 75 & 75 & 10 & 75 & 750 \\
\hline Subtotal & & & & 225 & & & 2250 \\
\hline \multicolumn{8}{|l|}{ LABORES CULTURALES } \\
\hline Aporque & Jornal & 3 & 75 & 225 & 30 & 75 & 2250 \\
\hline Deshierbe & Jornal & 7 & 75 & 525 & 70 & 75 & 5250 \\
\hline Tutorado & Jornal & 9 & 75 & 675 & 90 & 75 & 6750 \\
\hline Deschuponado & Jornal & 3 & 75 & 225 & 30 & 75 & 2250 \\
\hline Control fitosanitario & Jornal & 2 & 75 & 150 & 20 & 75 & 1500 \\
\hline Subtotal & & & & 1800 & & & 18000 \\
\hline COSECHA & Jornal & 5 & 75 & 375 & 50 & 75 & 3750 \\
\hline Subtotal & & & & 375 & & & 3750 \\
\hline TOTAL & & & & 3203.1 & & & 32031 \\
\hline
\end{tabular}

La estructura de los costos de producción ha sido tomada de Cuellar (2010), los datos han sido adaptados considerando la actualización de precios y la disponibilidad de materiales en el mercado local, la Tabla 3 permite conocer el detalle de los costos de producción para 500 $\mathrm{m}^{2}$ y $5000 \mathrm{~m}^{2}$.
Con la información obtenida se pudo elaborar la Tabla 4, donde se muestra ingreso por la producción en condiciones normales y con la implementación de los invernaderos. 


\section{Tabla 4}

Ingreso por la producción en condiciones normales y con la implementación de los Invernaderos tipo $A$ y $B$

\begin{tabular}{|c|c|c|c|c|}
\hline \multirow[b]{2}{*}{ Total superficie $\left(\mathrm{m}^{2}\right)$} & \multicolumn{3}{|c|}{ Ingreso esperado con la tecnología actual } & \multirow[b]{2}{*}{ Ingreso Total Bs. } \\
\hline & $\begin{array}{c}\text { Rendimiento } \\
\left(\mathrm{Kg} / \mathrm{m}^{2}\right)\end{array}$ & $\begin{array}{c}\text { Total } \\
\text { producción } \\
(\mathbf{K g .})\end{array}$ & $\begin{array}{l}\text { Precio } \\
\text { agricultor } \\
\text { (Kg.) }\end{array}$ & \\
\hline 500 & 1,2 & 600 & 4,85 & 2910 \\
\hline 5000 & 1,2 & 6000 & 4,85 & 29100 \\
\hline
\end{tabular}

\begin{tabular}{|c|c|c|c|c|}
\hline \multicolumn{5}{|c|}{ Ingreso esperado con la implementación de invernaderos } \\
\hline Total superficie $\left(\mathrm{m}^{2}\right)$ & $\begin{array}{c}\text { Rendimiento } \\
\left(\mathrm{Kg} / \mathrm{m}^{2}\right)\end{array}$ & $\begin{array}{l}\text { Total } \\
\text { producción } \\
(\mathbf{K g . )}\end{array}$ & $\begin{array}{l}\text { Precio } \\
\text { agricultor } \\
\text { (Kg.) }\end{array}$ & Ingreso Total Bs. \\
\hline \multicolumn{5}{|l|}{ Invernadero tipo A } \\
\hline 500 & 5 & 2500 & 3,85 & 9625 \\
\hline \multicolumn{5}{|l|}{ Invernadero tipo B } \\
\hline 5000 & 5 & 25000 & 3,85 & 96250 \\
\hline
\end{tabular}

Como se puede ver en la Tabla 4 los ingresos se incrementan considerablemente con la implementación de tecnología en el proceso productivo. Es decir, lo ingresos por la producción de tomate se incrementan en un $417 \%$.

\section{CONCLUSIONES}

La temática central de este artículo científico se refiere fundamentalmente a implementar un cambio tecnológico en los métodos productivos agrícolas en comunidades donde se sigue desarrollando procesos tradicionales con tecnologías que proveen baja productividad. La metodología productiva propuesta está siendo utilizada en otras partes del mundo con avances tecnológicos de última generación. En este caso particular se propone adaptar esta tecnología a la situación actual de los productores agrícolas del municipio de Sucre. De acuerdo con esta consideración se entiende que significa un salto importante en el uso de tecnológicas modernas que implica una necesidad de capacitación y acompañamiento por técnicos altamente capacitados en este sistema de producción. Asimismo, se considera que este cambio tecnológico va a inducir a los productores agrícolas a apropiarse de la tecnología y crear un sistema tecnológico moderno que aproveche las condiciones agroecológicas para beneficio de las características organolépticas de producción agrícola local.

Se propusieron 7 áreas fundamentales de acción: recursos naturales, recursos humanos, infraestructura y equipamiento, innovación tecnológica, capacitación y asistencia técnica, respeto al medio ambiente y cooperación institucional. El cumplimiento de la estrategia 
implica un trabajo sistemático y todas las áreas son fundamentales. Durante los primeros 5 años, la capacitación y asistencia técnica no podrán desarrollarse de manera adecuada si no existe cooperación institucional. Por otra parte, no se podrá garantizar el respeto al medio ambiente ni el éxito de la innovación tecnológica, si es que no se cuenta con capacitación y asistencia técnica. Es decir, el éxito de la estrategia en sus primeros años depende del éxito de cada una de las áreas de acción. Para posteriores años, la importancia de la cooperación institucional y la asistencia técnica reducirá considerablemente, porque los productores irán capitalizándose e irán ganando experiencia con el método de cultivo (invernadero), fertilización de la tierra, riego que evite estrés hídrico, ausencia de fenómenos meteorológicos adversos, uso de insumos mejorados.

En el mediano plazo se esperan beneficios directos e indirectos. Se beneficia de manera directa a los productores agrícolas con el incremento de su productividad, ya que, según el ejemplo expuesto, la producción de tomate podría incrementarse en un $417 \%$ manteniendo constante la extensión de tierra cultivada. Asimismo, al incrementarse los volúmenes de producción y la calidad de estos, se facilitará el comercio local y el ingreso a mercados nacionales. Entre los beneficiaros indirectos se tiene a los transportistas que trasladarán los productos a los diferentes mercados locales, los consumidores que adquirirán un producto ecológico y natural que no atente contra su salud, los comercializadores de insumos $y$, finalmente, se incrementan las opciones para las industrias de valor agregado, que a su vez aseguran el mercado para esta producción.

En el contexto de la Pandemia provocada por la COVID-19, la economía de Bolivia se ha visto afectada, golpeando fuertemente a todos los sectores productivos. La estrategia de producción agrícola tecnificada propuesta se constituye en una opción pertinente para elevar la calidad de vida de los productores agrícolas del municipio de Sucre y de la población en general. Se entiende que, al mejorar la economía de los productores, estos tendrán mejores posibilidades de educación, protección de sus familias y alimentación mejorada que les permita elevar sus defensas ante el virus SARS-CoV-2, otros virus y varias enfermedades. Asimismo, una vez implementada esta estratega, la experiencia servirá para motivar a otros municipios a implementar mejoras tecnológicas en sus procesos productivos.

\section{REFERENCIAS}

FAO. (2020). Organización de las Naciones Unidas para la Agricultura y la Alimentación World agriculture: towards 2015/2030

GADCH. (2018). Gobierno Autónomo Departamental de Chuquisaca, Proyecto: "Implementación de Huertos Urbanos Bajo Cubierta en la ciudad de Sucre" Agricultura Urbana y Periurbana

GAMS. (2015). Gobierno Autónomo Municipal de Sucre, Sucre Municipio Productivo https://sucre.bo/sucre-municipio-productivocentro-de-servicios-en-mecanizacionagricola-al-servicio-de-productores/

INE. (2015). Censo Agropecuario Bolivia 2013. Instituto Nacional de Bolivia. https://www.sudamericarural.org/images/en 
_papel/archivos/CENSO-AGROPECUARIOBOLIVIA_final.pdf

SENAMHI. (2020) Servicio nacional de Meteorología e Hidrología http://senamhi.gob.bo/index.php/sismet

SITAP. (2015). Sistema Integrado de Información Productiva, Informe Económico Productivo del Departamento de Chuquisaca https://siip.produccion.gob.bo/noticias/files /BI_2305202085291_INFCHQS2020.pdf

UASB. (2008). Convenio Marco de Colaboración entre la Universidad de Cádiz, España y la Universidad Andina Simón Bolívar, Bolivia. Cádiz

UASB. (2016). Universidad Andina Simón Bolívar. Análisis económico del cultivo de tomate cherry bajo invernadero en dos comunidades del distrito 8 del municipio de Sucre

UCM. (2020). Revista electrónica de Medio Ambiente UCM. LA AGRICULTURA INTENSIVA DEL PONIENTE ALMERIENSE. Diagnóstico e instrumentos de gestión ambiental

Urresterazu, M. (2000). Manual de cultivo sin suelo. Grupo Mundi Prensa; Almería 2000

Valera D. L. M.; Molina, F. D. A. \& Gil, J. A. R. (2000). Los invernaderos de Almería: tipología y mecanización del clima (Vol. 9). Universidad Almería. 